\title{
Research on Visual Rid and Path Planning of Industrial Robot
}

\author{
Jingyun Zhao ${ }^{1}$ \\ Department of Mechanical Engineering, Henan \\ Mechanical and Electrical Engineering College, \\ Xinxiang, Henan 453002, China \\ e-mail: hncad@163.com
}

\author{
Pengfei Wang ${ }^{2}$ \\ Xijing University, Xi'an, 710123, China \\ e-mail:315705804@qq.com
}

\begin{abstract}
Traditional path planning algorithm for multiobjective circumstances, primarily on a single multiinformation fusion Select avoidance path, in the scale of sludge entangled region vulnerable to blind, unable to robot path sludge entangled environment for accurate planning. This paper presents an improved robot vision route planning method to get rid of, improve the ability of the robot's environment entangled in the mud of the robot around obstacles. To this end, an improved robot path planning method to get rid of visual entangled, with the robot vision instrument collection features sludge entangled with a normalization method to integrate visual information into the programming model to choose the best path, the robot get rid of sludge entanglement and the shortest route requires integration into a fitness function, get the best robot rid of paths through the genetic algorithm search. Experimental results show that the method of sludge entanglement under ambient robot path planning to get rid of lengths and efficiency are superior to the traditional model, with high robustness.
\end{abstract}

Keywords-Friction coefficient; ANSYS; contact analysis; neural network; genetic algorithm

\section{INTRODUCTION}

Mobile robot path planning problem is an obstacle in the case, to find a way to ensure that the robot can bypass obstacles in the shortest possible time. With the gradual expansion of robotic applications to seek a reasonable approach to the robot is entangled state is planning to get rid of a failed path accurately, become a hot issue related academic research [1-4]. Path planning algorithm is not the traditional method of multi-objective analysis of the situation, the main basis for selecting a single message collision path, the path to get randomness large and medium-sized sludge entangled in the area vulnerable to blind, unable to sludge entangled environment accurate robot path planning $[5,6]$.

Industrial robots are robotic device automatically performs the work, is to rely on their own power and control capabilities to achieve the various functions of a machine [7]. It can accept human command, you can run the program in accordance with pre-programmed, the principles of the program of Action of modern industrial robots can also be developed in accordance with artificial intelligence techniques [8-11]. This article describes the basic components of industrial robots, especially industrial robots core controller, the controller is proposed to solve the problem of the reliability of accuracy, and gives relevant solutions and strategies.

Robots can help replace humans in many ways, even complex, dangerous and repetitive work, since the development, application of robots will continue to be reflected, but generally work in industrial robots in harsh working environments, we need shock proof, dust, electromagnetic interference, to ensure reliable operation of industrial robots. Such as: "Minimum effort and proportion of" allocation method is suitable for the first time developed, have been put into use and is ready for production, and further improve the reliability of its industrial robot system; "hybrid" distribution method is a simple industrial robot system reliability allocation methods, this method is suitable for the development of new industrial robots were when its distribution system reliability in the absence of constraints, so that the development of industrial robots with higher reliability; "two-tier optimization" allocation It is in a constrained condition under optimum reliability allocation methods. These three industrial robot Allocation Policy as its reliability is a key part of the design, not only theoretical research value, but also has great practical significance.

\section{VISUAL RID FAULT PATH SELECTION}

Robot Calibration is a set of kinematic parameters error modeling, robot end attitude error measurement and robotics actual parameter identification in one process [12]. Parameter identification is measured robot end attitude error identification parameters important part. In order to improve the accuracy of the robot motion, parameters can be used as a two-step linear error recognition error tracking error and positioning error separation. The use of relatively rigid body rotation error identified only kinematic parameters associated with the rotation error; the calibrated parameters and error identified from the use of rigid body kinematic parameters remaining errors. Not only to avoid the two-step identification method transfer measurement coordinate system and the robot coordinate system conversion, and parameter estimation difference, and effective solution to the singular and nonlinear problems that exist in the distance method, you can quickly find linear solution parameter errors. To describe the case of path planning environment entangled sludge robot, the robot's work space set as shown in Fig. 1. Shaded area which described 
obstacle may be entangled sludge environmental information using neural network model shown in Fig. 1 will be described.

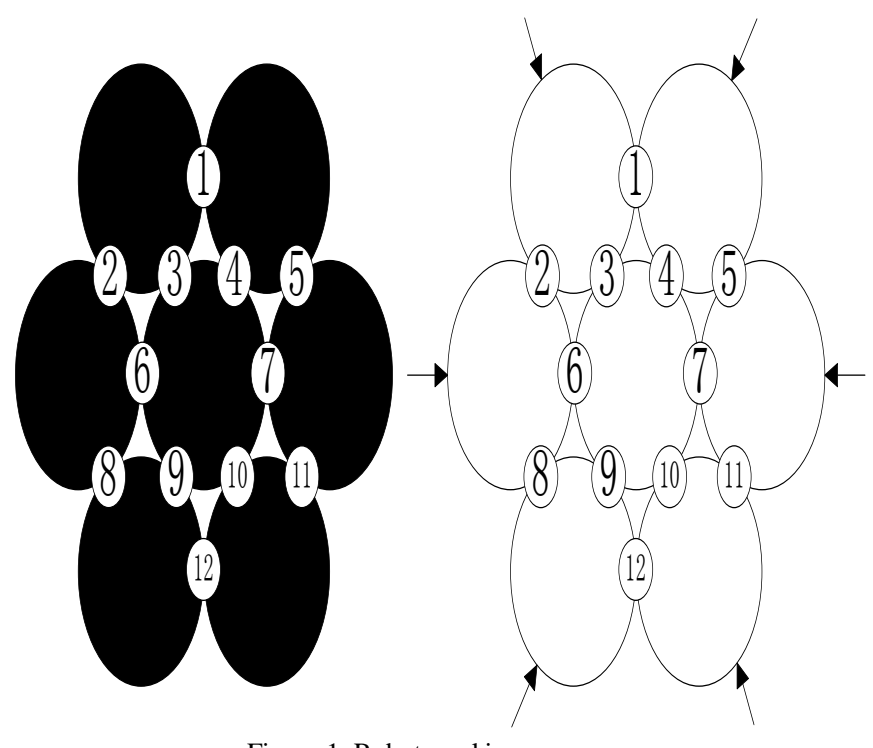

Figure 1. Robot working space

Genetic algorithm is a global optimization method to imitate biological nature, which can be processed multiple feasible solutions to avoid the emergence of local optimum, and the like by the mutation operator to optimize the feasible solutions to generate new viable solution, eventually produce global optimal solution. It has strong adaptability and robustness, for selecting the best path. Because of the differential operator (1) and left (2) equivalent differential operator left, so we introduce three order Green function tensor:

$$
\sum\left(\mathbf{x} \mid \mathbf{x}^{\prime}, \omega\right)=\lambda \mathbf{I} \nabla[\mathbf{G}+\mu(\nabla \mathbf{G}+\mathbf{G} \nabla)
$$

Or use the subscript simplified form:

$$
\sum_{i n v}=\lambda \delta_{l m} \partial_{k} G_{k m}+\mu\left(\partial_{j} G_{m n}+\partial_{m} G_{\mathrm{ln}}\right)
$$

Here $\partial_{m}=\partial / \partial x_{m}$,(10) was also presented as:

$$
\nabla \square \sum\left(\mathbf{x} \mid \mathbf{x}^{\prime}, \omega\right)+\rho \omega^{2} \mathbf{G}\left(\mathbf{x} \mid \mathbf{x}^{\prime}, \omega\right)=-\delta\left(\mathbf{x}-\mathbf{x}^{\prime}\right) \mathbf{I}
$$

Here on behalf of $\mathbf{x} \mid \mathbf{x}^{\prime}, \omega$ stress, three vertical force produced at the point $\mathrm{x}$ and $\mathbf{x} \mid \mathbf{x}^{\prime}, \omega$ T's relationship with $G$ and $u$ in the same, we call the $G$ displacement Green's function tensor, called Green Stress function tensor. Industrial robots face a different application of technology in industrial environments and the use of a variety of requirements. It is possible to use one of the working environment to a variety of robotics applications such as the use of visual technology, applications for multi-industrial robot control, voice recognition and other aspects. Some specific working environment will also use some special techniques, such as fuzzy control, neural network control technology, some communications technologies. Here, only two commonly used to introduce robotics. The algorithm of GA was shown in Fig. 2.

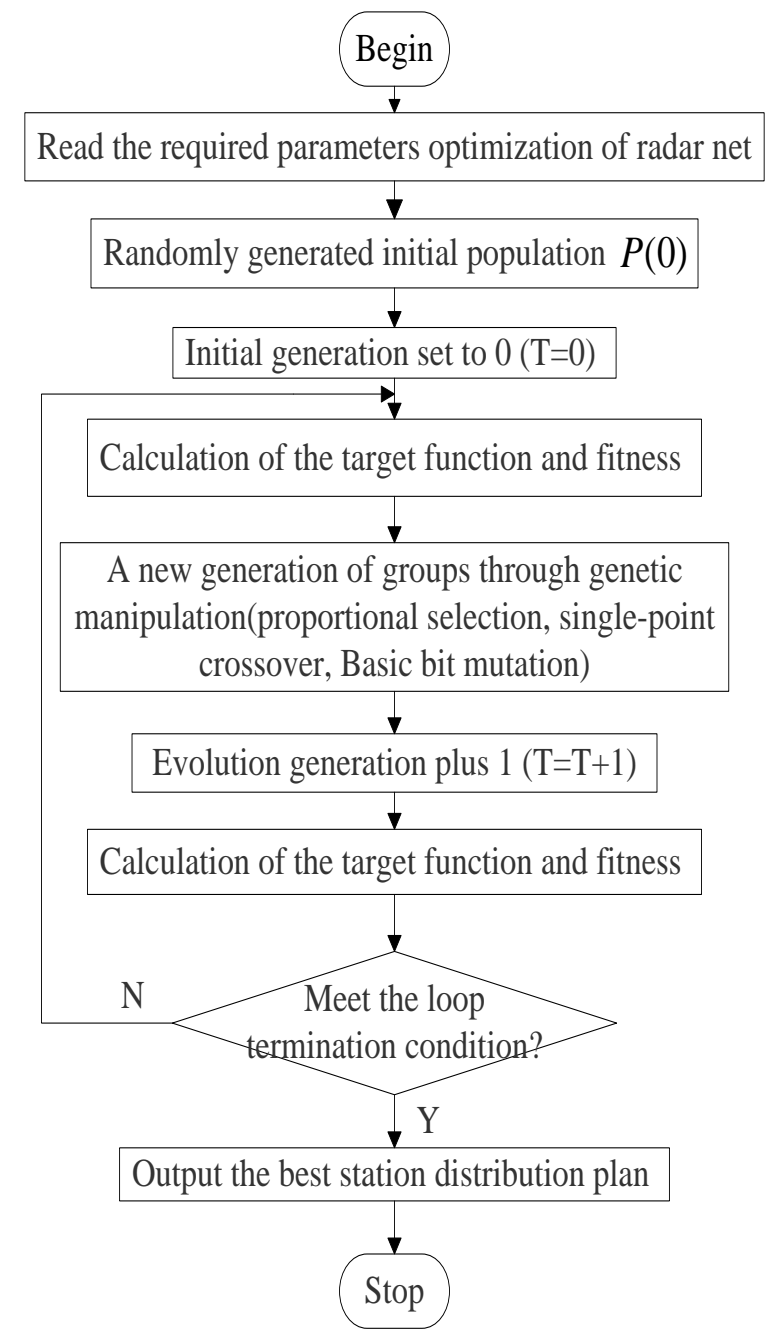

Figure 2. The algorithm of GA

\section{INDUSTRIAL ROBOTS CONTROL TECHNOLOGY}

\section{A. The contact finite element analysis method}

Robots get rid of the entanglement zone path mostly two-dimensional coding to achieve. If converted into a one-dimensional code, it will calculate the convenience, and improve the operating efficiency of the algorithm. In order to reduce costs, but also in order to save space, and sometimes requires the use of an industrial robot arm operates two or more stations in the same minute, the arm can be more than one, but the controller and other peripherals only set, and require the robot's work is not affected. dl:

Analysis of the rotation angle of the spiral line alpha

$$
d l=\sqrt{d x^{2}+d y^{2}+d z^{2}}=\sqrt{r^{2}+\left(\frac{L}{2 \pi}\right)^{2}} d t
$$

Then:

$$
\frac{d l}{d t}=\frac{\sqrt{4 \pi^{2} r^{2}+L^{2}}}{2 \pi}
$$

The line angle: 


$$
\begin{gathered}
\sin \alpha=\frac{L / 2 \pi}{\sqrt{r^{2}+(L / 2 \pi)^{2}}}=\frac{L}{\sqrt{4 \pi^{2} r^{2}+L^{2}}} \\
\cos \alpha=\frac{2 \pi r}{\sqrt{4 \pi^{2} r^{2}+L^{2}}}
\end{gathered}
$$

The robot arm and controller signal line can only have a set, you must use the same set of I/O signal line is configured for both arms. Thus, you can know more industrial robot control for production line applications technology high real-time requirements of the system, to coordinate the various control objects, so you can use the embedded system may deprive type, except in addition to a unified security communication bus technology.

\section{B. Industrial robot trajectory optimization strategy}

For the motion of an industrial robot, often it wants its shortest trajectory, use the shortest time. Therefore, the industrial robot controller can be time-optimal trajectory planning and control. We need to ensure that within the constraints of the robot joint displacement, velocity, acceleration, and second-order boundary value acceleration, the robot hand in Cartesian space along a predetermined path of movement of the shortest time. Thus the need for relevant strategies, such as the planned joint trajectory in the form of quadratic polynomial plus cosine function, not only can ensure the joint movement of displacement, velocity and acceleration in a row, but also ensure that all the articulation of second-continuous acceleration. In this way, not only can improve the efficiency of the robot and can extend the working life of the robot. Experiments show that the use of effective trajectory optimization strategy for industrial robots under kinematic constraints can achieve optimal trajectory and movement time shortest distance, and ensure the reliability of the robot movement.

\section{EXPERIMENT AND RESULTS}

The proposed method and the traditional method of sludge entangled environment under dynamic obstacle avoidance path simulation result, as shown in Fig. 3 respectively. Wherein the circle represents the start and end points, sludge entangled area is represented by a rectangular box. Two different sludge entangled area, the robot along the vertical direction to zero. Speed of $2 \mathrm{~m} / \mathrm{s}$ in the horizontal direction and 0 . Speed of $18 \mathrm{~m} / \mathrm{s}$ of onward robot current real-time speed is zero. $24 \mathrm{~m} / \mathrm{s}$. Experimental results show that: the algorithm can be entangled environment sludge accurate robot path planning, can effectively avoid the associated sludge entangled area and a lot of planning path length is smaller than the traditional method; When traditional methods of planning, there will be entangled area with sludge or intersection collision problems, can not plan a reasonable obstacle Avoidance path.

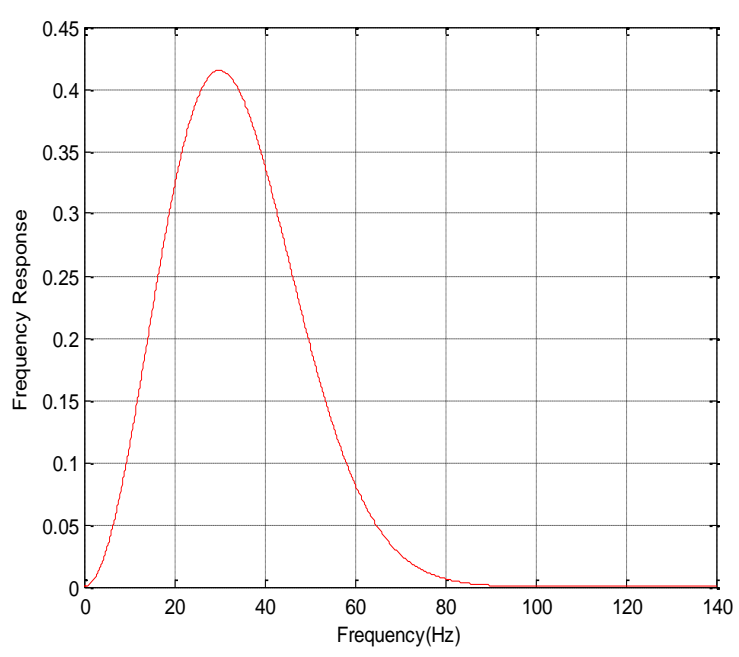

Figure 3. The obstacle avoidance path simulation result

Adopt appropriate protective measures and corresponding strategies, efficient and accurate completion of the robot corresponding control. Since the industrial robot system reliability distribution is its integral part reliability design, so when optimizing the design of industrial robots, on the allocation method System Reliability Index research has important theoretical and practical significance, and based on this, you can different system requirements in the context of an industrial robot system reliability configuration policies.

Machine vision systems are now widely used in the field of visual guidance and automated assembly visual inspection, robot. The vast majority of the visual scene information feedback is applied to the target in the scene to identify, locate and motion analysis, vision-based targeting can be understood as the following three conditions: selfpositioning of the robot, actually captured the target, and the manipulator between the relative positioning and target. In addition to establishing a single robot simple visual feedback or a single stand-alone vision system feedback, but can be used more cooperative industrial robot vision system that can enhance their coordination and cooperation and the sharing of resources and give full play to the overall advantages of multi-robot systems. Use visual feedback of information systems, industrial robots may make an appropriate judgment based on the current situation, effective control of the target object.

\section{CONCLUSIONS}

Current industrial robots increasingly rapid development, the factory mechanization and automation is inevitable. In modern industrial production, industrial robots automated production line equipment has become the mainstream and the future direction of automation equipment. Automotive industry, electronic industry, engineering machinery and other industries has been widely used industrial robots automated production lines, in order to ensure product quality, increase productivity, and avoid a lot of accidents. Industrial robots are widely used in production to promote the development of robot technology, the robot to complete the task can be more accurate and efficient. Thus, resulting in a lot of control 
technology and robotics, but their core is the controller, enabling the system to run efficiently and reliably.

\section{REFERENCES}

[1] Luo Xue-hui, Yang Ye, Li Xia, "Modified Shuffled Frog-Leaping Algorithm to Solve Traveling Salesman Problem", Journal on Communications, vol.30,no.7, pp.130-135, 2009.

[2] Zhang Xiaodan, H u Feng, Zhao Li, "Improved Shuf F Led Frog Leaping Algorithm Based On Molecular Dynamics Simulations", Journal of Data Acquisition \& Processing, vol.27, no.3, pp.327-332, 2012.

[3] Gg Yu, Wang Xueping, Liang Jing, "Improved Shuffled Frog Leaping Algorithm", Journal of Computer Applications, vol.32, no.1, pp.234-237, 2012.

[4] Huynh T H, "A Modified Shuffled Frog Leaping Algorithm For Optimal Tuning of Multivariable PID Controllers ", IEEE Int Conf on Industrial Technology,pp.1-6, 2008.
[5] Man Cheol Kim,Jinkyun Park, Wondea Jung, Hanjeom Kim, Yoon Joong Kim. Development of a standard communication protocol for an emergency situation management in nuclear power plants $[\mathrm{J}]$. Annals of Nuclear Energy,2010,37(6): 888-893.

[6] Majid Khodier, Gamcel Saleh. Beamforming and Power control for interference reduction in wireless communications using particle swarm optimization[J]. International Journal of Electronics and Communications, 2010,64(6):489-502.

[7] Aleksandar Radonjie, Vladimir Vujicie. Integer SEC-DED codes for low power communications[J]. Information Processing Letters, 2010,110(12-13):518-520.

[8] Zhang S, Yao H, Sun X, et al. Sparse coding based visual tracking: Review and experimental comparison [J]. Pattern Recognition, 2013, 46(7): 1772-1788.

[9] Yang H, Shao L, Zheng F, et al. Recent advances and trends in visual tracking: A review [J]. Neurocomputing, 2011, 74(18): 38233831.

[10]Chen J, He Y,Wang J. Multi-feature fusion based fast video flame detection [J], Building and Environment, 2010,45(5): 1113-1122. 